\title{
Comment on "Web-Based Measure of Life Events Using Computerized Life Events and Assessment Record (CLEAR): Preliminary Cross-Sectional Study of Reliability, Validity, and Association With Depression": Validity and Methodological Issues
}

Jamal Rahmani ${ }^{1}$, MSc, PhD; Roya Karimi ${ }^{2}$, MSc, PhD; Farideh Mohtasham ${ }^{3}$, MSc, PhD; Siamak Sabour ${ }^{2,4}$, MD, PhD

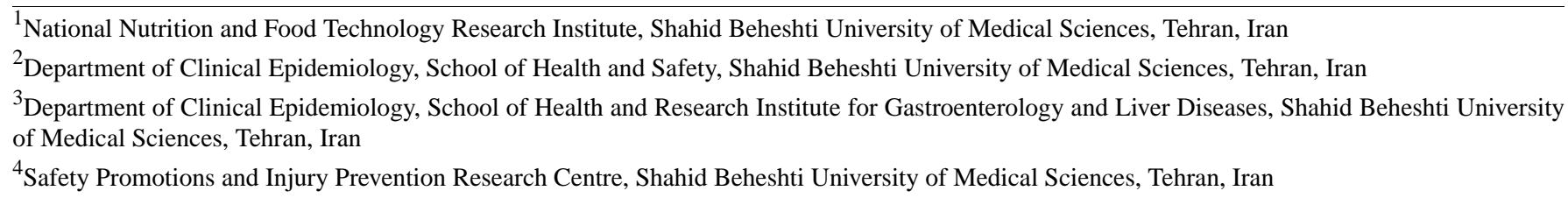

Corresponding Author:

Siamak Sabour, MD, PhD

Department of Clinical Epidemiology

School of Health and Safety

Shahid Beheshti University of Medical Sciences

Chamran Highway

Tehran

Iran

Phone: 982122421814

Email: s.sabour@sbmu.ac.ir

\section{Related Articles:}

Comment on: https://mental.jmir.org/2019/1/e10675/

Comment in: https://mental.jmir.org/2020/5/e15434/

(JMIR Ment Health 2020;7(5):e14505) doi: 10.2196/14505

\section{KEYWORDS}

validity; methodological issues; diagnostic test

We were interested in the article titled, "Web-Based Measure of Life Events Using Computerized Life Events and Assessment Record (CLEAR): Preliminary Cross-Sectional Study of Reliability, Validity, and Association With Depression" published in JMIR Mental Health [1].

One of the aims of the abovementioned study was to assess the validity of Computerized Life Events and Assessment Record (CLEAR), considering the Life Events and Difficulties Schedule (LEDS) and the List of Threatening Experiences Questionnaire (LTE-Q) as gold standards among 328 participants (126 students; 202 matched midlife sample: 127 unaffected controls, 75 recurrent depression cases). The authors concluded that CLEAR has acceptable validity and great potential for effective use in research and clinical practice. However, there are some methodological issues in this conclusion that are mentioned below.

First, there are some measures that can be applied to the assessment of the validity of a test including sensitivity, specificity, positive predictive value, negative predictive value, positive likelihood ratio (LR+; ranging from 1 to infinity; the higher the $\mathrm{LR}+$, the more accurate is the test), negative likelihood ratio (LR-; ranging from 0 to 1 ; the lower the LR-, the more accurate is the test), and odds ratio (ratio of true to false results) [2-5]. According to the results, sensitivity of CLEAR was $59.1 \%$ and $43.1 \%$ compared to LEDS and LTE-Q, respectively, as gold standards. Likewise, specificity of CLEAR was $65.4 \%$ and $78.6 \%$, respectively, compared to the abovementioned gold standards.

It is good to know that sensitivity is an important measure in public health aspects instead of clinical fields. Likewise, the positive predictive value and negative predictive value are among measures that are more appropriate for advice about the validity of a diagnostic test for clinical purposes [3-5]. Therefore, we suggest applying predictive values, likelihood ratios, odds ratio, and diagnostic accuracy to decide the validity of CLEAR. Moreover, according to the data of study, LR+, LR-, odds ratio, and diagnostic accuracy of CLEAR will be $1.6,0.6,2.6$, and $62 \%$, respectively, compared to LEDS (Tables 1 and 2 ) and $1.9,0.7,2.6$, and $60 \%$, respectively compared to 
LTE-Q (Tables 3 and 4). Therefore, there is a high level of insufficient evidence to conclude that the validity of the CLEAR uncertainty for decisions based on these values, and there is test is acceptable.

Table 1. Two by two table of Computerized Life Events and Assessment Record compared to Life Events and Difficulties Schedule as the gold standard.

\begin{tabular}{llll}
\hline CLEAR $^{\mathrm{a}}$ & \multicolumn{2}{c}{ LEDS $^{\mathrm{b}}$ (gold standard) } & \\
& Positive & Negative & Total \\
\hline Positive & 59 & 35 & 94 \\
Negative & 41 & 65 & 106 \\
Total & 100 & 100 & 200 \\
\hline
\end{tabular}

${ }^{a}$ CLEAR: Computerized Life Events and Assessment Record.

bLEDS: Life Events and Difficulties Schedule.

Table 2. Assessing the validity of Computerized Life Events and Assessment Record compared to Life Events and Difficulties Schedule as the gold standard.

\begin{tabular}{ll}
\hline Parameter & Estimate \\
\hline Sensitivity (\%) & 59 \\
Specificity (\%) & 65 \\
Positive predictive value (\%) & 63 \\
Negative predictive value (\%) & 61 \\
Diagnostic accuracy (\%) & 62 \\
Likelihood ratio of a positive test & 1.6 \\
Likelihood ratio of a negative test & 0.6 \\
Diagnostic odds & 2.6 \\
\hline
\end{tabular}

Table 3. Two by two table of Computerized Life Events and Assessment Record compared to List of Threatening Experiences Questionnaire as the gold standard.

\begin{tabular}{llll}
\hline CLEAR $^{\mathrm{a}}$ & LTE-Q & \\
& Positive & & \\
& Told standard) & Negative & 65 \\
\hline Positive & 43 & 22 & 135 \\
Negative & 57 & 78 & 100 \\
Total & 100 & 100 & 200 \\
\hline
\end{tabular}

${ }^{\mathrm{a} C L E A R: ~ C o m p u t e r i z e d ~ L i f e ~ E v e n t s ~ a n d ~ A s s e s s m e n t ~ R e c o r d . ~}$

${ }^{\mathrm{b}}$ LTE-Q: List of Threatening Experiences Questionnaire.

Table 4. Assessing the validity of Computerized Life Events and Assessment Record compared to List of Threatening Experiences Questionnaire as the gold standard.

\begin{tabular}{lc}
\hline Parameter & Estimate \\
\hline Sensitivity (\%) & 43 \\
Specificity (\%) & 78 \\
Positive predictive value (\%) & 66 \\
Negative predictive value (\%) & 58 \\
Diagnostic accuracy (\%) & 60 \\
Likelihood ratio of a positive test & 1.9 \\
Likelihood ratio of a negative test & 0.7 \\
Diagnostic odds & 2.6 \\
\hline
\end{tabular}




\title{
Conflicts of Interest
}

None declared.

\section{References}

1. Bifulco A, Spence R, Nunn S, Kagan L, Bailey-Rodriguez D, Hosang GM, et al. Web-Based Measure of Life Events Using Computerized Life Events and Assessment Record (CLEAR): Preliminary Cross-Sectional Study of Reliability, Validity, and Association With Depression. JMIR Ment Health 2019 Jan 08;6(1):e10675 [FREE Full text] [doi: 10.2196/10675] [Medline: 30622088]

2. Szklo M, Nieto J. Epidemiology: beyond the basics. 3rd ed. Manhattan, NY: Jones and Bartlett Learning; 2014.

3. Sabour S, Ghassemi F. The validity and reliability of a signal impact assessment tool: statistical issue to avoid misinterpretation. Pharmacoepidemiol Drug Saf 2016 Oct;25(10):1215-1216. [doi: 10.1002/pds.4061] [Medline: 27696610]

4. Sabour S. Validity and reliability of the new Canadian Nutrition Screening Tool in the 'real-world' hospital setting: Methodological issues. Eur J Clin Nutr 2015 Jul;69(7):864. [doi: 10.1038/ejcn.2015.69] [Medline: 25920428]

5. Sabour S. Validity and reliability of the 13C-methionine breath test for the detection of moderate hyperhomocysteinemia in Mexican adults; statistical issues in validity and reliability analysis. Clin Chem Lab Med 2014 Dec;52(12):e295-e296. [doi: 10.1515/cclm-2014-0453] [Medline: 24933629]

\section{Abbreviations \\ CLEAR: Computerized Life Events and Assessment Record \\ LEDS: Life Events and Difficulties Schedule \\ LR-: negative likelihood ratio \\ LR+: positive likelihood ratio \\ LTE-Q: List of Threatening Experiences Questionnaire}

\author{
Edited by J Torous; submitted 26.04.19; peer-reviewed by A Bifulco; accepted 10.07.19; published 21.05.20 \\ Please cite as: \\ Rahmani J, Karimi R, Mohtasham F, Sabour S \\ Comment on "Web-Based Measure of Life Events Using Computerized Life Events and Assessment Record (CLEAR): Preliminary \\ Cross-Sectional Study of Reliability, Validity, and Association With Depression”: Validity and Methodological Issues \\ JMIR Ment Health 2020;7(5):e14505 \\ URL: https://mental.jmir.org/2020/5/e14505 \\ doi: $10.2196 / 14505$ \\ PMID: $\underline{32436853}$
}

(CJamal Rahmani, Roya Karimi, Farideh Mohtasham, Siamak Sabour. Originally published in JMIR Mental Health (http://mental.jmir.org), 21.05.2020. This is an open-access article distributed under the terms of the Creative Commons Attribution License (https://creativecommons.org/licenses/by/4.0/), which permits unrestricted use, distribution, and reproduction in any medium, provided the original work, first published in JMIR Mental Health, is properly cited. The complete bibliographic information, a link to the original publication on http://mental.jmir.org/, as well as this copyright and license information must be included. 